\title{
BMJ Quality Choosing Wisely: A Quality Improvement Initiative to Decrease Unnecessary Preoperative Testing
}

\author{
John Matulis, Stephen Liu, John Mecchella, Frederick North, Alison Holmes
}

To cite: Matulis J, Liu S, Mecchella J, et al. Choosing Wisely: A Quality Improvement Initiative to Decrease Unnecessary Preoperative Testing. BMJ Quality Improvement Reports 2017;6:bmjqir.u216281. w6691. doi:10.1136/ bmjquality.u216281.w6691

CrossMark

Dartmouth-Hitchcock Medical Center, USA

\section{ABSTRACT}

Dartmouth-Hitchcock Medical Center is a rural, academic medical center in the northeastern United States; its General Internal Medicine (GIM) division performs about 900 low and intermediate surgical risk preoperative evaluations annually. Routine preoperative testing in these evaluations is widely considered a lowvalue service. Our baseline data sample showed unnecessary testing rates of approximately $36 \%$. A multi-disciplinary team used a micro-systems approach to analyze the existing process and formulate a rapid cycle improvement strategy. Our improvement efforts focused on implementation of a Nurse Practitioner and Physician Assistant (Associate Provider) clinic to incorporate standardized protocols for preoperative assessment. Plan-Do-Study-Act (PDSA) cycles included creation of a dedicated Associate Provider run preoperative clinic, modifying and operationalizing a scheduling scheme, and creating and implementing Electronic Health Record (EHR) tools. We used Statistical Process Control (SPC) methods to analyze time ordered data for the usual care process and to compare performance with the novel preoperative clinic. The Associate Provider preoperative clinic showed unnecessary testing rates of $4 \%$ compared with $23 \%$ in the usual care cohort $(p<.001)$ within 3 months of implementation. When testing rates across the entire division were analyzed, there was no significant change. In our GIM division this preoperative clinic was effectively staffed with Associate Providers. Dedicated leadership support, incorporating input from a diverse improvement team, and balancing innovation with other clinical needs are important elements for success. We hypothesize that protecting clinical time to focus on preoperative care, monitoring and modifying scheduling processes, and improving support for electronic health record tool implementation would have yielded further performance improvements. Our experience provides valuable learning for other primary care practices with similar challenges. Identifying appropriate patients for inclusion in these clinic visits while optimizing primary care provider collaboration are important future challenges.

\section{PROBLEM}

Unsustainable healthcare costs and wasteful provision of services are well recognized in
American healthcare ${ }^{1}$. It is estimated that $\$ 18$ billion (U.S.) is spent annually on preoperative testing, although how much is wasteful remains unknown ${ }^{2}$. To understand the scope of this problem at our own institution, a 3 month chart review was performed. Using a methodology grounded in clinical guidelines, we established that $36 \%$ of patients receiving preoperative care in our clinic were receiving unnecessary testing (Table 1).

The aim of this quality improvement project was to decrease rates of unnecessary preoperative testing in patients undergoing low-risk surgery. More specifically, we intended to ${ }^{1}$ reduce the rate of unnecessary preoperative testing across our division, defined by published guidelines, in patients undergoing preoperative assessment from $36 \%$ to $10 \%$ over a 9 month period $\mathrm{and}^{2}$ reduce the average appointment duration for preoperative history and physicals from 48 minutes to 35 minutes over the same period.

Our General Internal Medicine (GIM) clinic is a part of an academic, tertiary care center and is staffed by 16 staff physicians, 6 Associate providers (Nurse Practitioner and Physician Assistant), and 39 residents. The GIM section performs approximately 900 pre-operative evaluations annually. The clinic and medical center have used the Electronic Health Record (EHR) Epic (Epic Systems Corporation, Verona, WI), since 2011.

\section{BACKGROUND}

Background: Both Medicare and the Joint Commission mandate that patients scheduled for surgical procedures have a completed History and Physical performed within 30 days of the procedure ${ }^{3}$. As early as the late $1970 \mathrm{~s}$, well-designed randomized controlled trial called into question the practice of preoperative testing prior to elective surgery $^{4}$. Multiple studies have replicated this 
Table 1 Baseline rates of unnecessary* preoperative testing

\begin{tabular}{|c|c|c|c|c|c|c|c|}
\hline Surgery & $\begin{array}{l}\text { Total } \\
\text { Visit } \\
\text { Count }\end{array}$ & $\begin{array}{l}\% \text { of visits with } \\
\text { unnecessary } \\
\text { preoperative testing } \\
\text { (count) }\end{array}$ & $\begin{array}{l}\% \text { with } \\
\text { Complete } \\
\text { blood count }\end{array}$ & $\begin{array}{l}\% \text { with } \\
\text { Metabolic } \\
\text { panels }\end{array}$ & $\begin{array}{l}\text { \% with } \\
\text { EKG }\end{array}$ & $\begin{array}{l}\% \text { with } \\
\text { Chest } \\
\text { X-ray }\end{array}$ & $\begin{array}{l}\% \text { with } \\
\text { other } \\
\text { testing }\end{array}$ \\
\hline Cataracts & 60 & $30 \%(18)$ & $17 \%(10)$ & $13 \%(8)$ & $18 \%(11)$ & $2 \%(1)$ & $2 \%(1)$ \\
\hline $\begin{array}{l}\text { All Low Risk } \\
\text { (include } \\
\text { cataract) }\end{array}$ & 106 & $33 \%(35)$ & $15 \%(7)$ & $13 \%(6)$ & $24 \%(11)$ & $2 \%(1)$ & 0 \\
\hline $\begin{array}{l}\text { Intermediate } \\
\text { Risk }\end{array}$ & 97 & $40 \%(39)$ & $24 \%(23)$ & $21 \%(20)$ & $28 \%(27)$ & $5 \%(5)$ & $8 \%(8)$ \\
\hline All & 203 & $36 \%(73)$ & $22 \%(44)$ & $18 \%(37)$ & $28 \%(57)$ & $3 \%(7)$ & $4 \%(9)$ \\
\hline
\end{tabular}

finding, including several additional randomized control trials and two systematic reviews. ${ }^{5}$ Despite strong evidence that performing diagnostic testing such as laboratory tests, electrocardiograms (EKG) and chest x-rays provide limited benefit in routine preoperative care, this practice remains widespread. ${ }^{6}$

This significant body of evidence eventually led to professional societies, notably the American Society of Anesthesia since 2002, to recommend that performance of routine laboratory evaluations in asymptomatic preoperative patients be curtailed. ${ }^{7}$ The Choosing Wisely Campaign $^{\mathrm{TM}}$ aims to foster discussion between patients and providers about services which provide questionable value; seven specialty societies participating in Choosing Wisely have recommended against performing routine preoperative testing prior to low-risk surgery. ${ }^{8}$

Baseline data collected by chart abstraction showed that $36 \%(73 / 203)$ of all patients who had a preoperative evaluation within our primary care division received unnecessary testing (Table 1). This baseline data was abstracted by a single reviewer (JCM) analyzing consecutive preoperative evaluations performed in our GIM clinic between the dates of July 1st 2013 and September 1st 2013. Smaller samples of monthly data after this time were collected to assess for unrelated temporal trends. In our division all high-risk surgical cases (predominantly vascular and intra-thoracic) are evaluated in the section of cardiology through a separate process; both baseline and subsequent data collections excluded any evaluations of patients undergoing high-risk surgery.

The majority of surgical sections at our institution do not perform their own preoperative evaluations, thus the measured preoperative testing is under the control of the GIM provider evaluating the patient. Depending on the surgeon's preference, a limited number of evaluations are performed through an institutional preadmission testing center staffed by clinicians from the Section of Anesthesiology; these evaluations were not included as part of our analysis. We were not aware of any co-occurring changes in the surgical or anesthesia preoperative care processes which affected the quantity or complexity of patients seen in GIM during this time period.

\section{BASELINE MEASUREMENT}

Outcome measures were proportion of preoperative patients receiving an unnecessary test, and duration of scheduled appointment time. We procured data for review by querying a central EHR data repository, and the first author (JCM) manually reviewed relevant charts to verify abstracted data and to assess concordance of ordered tests with published guidelines. Scheduled visit length was provided through an EHR query.

We used the patient visit as the unit of analysis. Monthly, we measured unnecessary test rates, numbers of unnecessary tests and scheduled visit times, and plotted them on statistical process control (SPC) charts in weekly time intervals. We analyzed dichotomous data by comparing the number of patients receiving at least one unnecessary test to all studied patients. These rates were then plotted on proportion charts ( $p$-charts) which showed the proportion of patients receiving unnecessary tests over time; count data was plotted using xMR charts. We used an Analysis of Means (ANOM) to compare testing rates between the preoperative clinic and usual care. Comparative analyses between the preoperative clinic (dedicated Associate Provider intervention clinic) and usual care (non-intervention preoperative visits) were performed using Chi-squared or student's t-test as appropriate. Statistical analyses were performed using the statistical software QI macros, 2013 (KnowWare Intl, Denver CO) and JMP, version 10.0 (SAS Corporation, Cary, NC).

\section{DESIGN}

Using a microsystems approach based off the Institute for Healthcare Improvement (IHI) model for Improvement, ${ }^{9}$ we generated pre-intervention process maps following direct observation of clinic processes and 
cause-and-effect diagrams (supplementary files) using provider interviews from a convenience sample. This pre-work helped us to determine local factors contributing to unnecessary testing. Our improvement team, through the creation of the cause and effect diagram concluded that important contributory factors to unnecessary testing, in order of modifiability included: ${ }^{1}$ practice tradition ${ }^{2}$ lack of familiarity with current guidelines and institutional preoperative requirements $\mathrm{and}^{3}$ time constraints. Pre-intervention, we found no standardized process for performing the preoperative evaluation, nor in the ordering of preoperative tests. Although institutional guidance on preoperative documentation was available, providers were unaware of this resource. All preoperative ordering was under complete discretion of the individual provider.

Using this information, we developed a standardized process for pre-operative visits to address clinical knowledge gaps and time constraints. Creating an Associate provider (Nurse Practitioner and Physician Assistant) run preoperative clinic as a mode of providing preoperative care addressed several underlying contributory factors. Many Associate providers in our division were in the earlier parts of their careers and thus the practice tradition component was felt to be more easily modifiable; additionally, there was significantly greater clinical access and scheduling flexibility among associate providers compared to primary care physicians (PCPs).

\section{STRATEGY}

The project team began performing PDSA cycles in the summer of 2014.

During PDSA cycle 1 we recruited Associate Providers to staff the preoperative clinic. An enabling factor in implementation was that the clinic faced significant access challenges. We hypothesized that by scheduling preoperative visits with Associate providers, instead of the patient's PCP, clinic access for PCPs would also be improved.

The second PDSA cycle focused on establishing the preoperative clinic scheduling process. The novel preoperative clinic required altering existing clinic flow, scheduling and resource allocation. We studied scheduling flow carefully and implemented multiple educational sessions for the scheduling staff; additional education sessions were required due to staff turnover. Ultimately, operationalizing these clinic schedules required us to "hold" clinic appointment slots for preoperative evaluations which if unfilled could subsequently be opened within 48 hours of the appointment for other visits.

The third PDSA cycle focused on implementation and optimization of the newly designed preoperative clinic. Patients scheduling a preoperative evaluation were initially offered an appointment with either their PCP, another provider within their care team, or the preoperative clinic. However, due to low fill rates, a new scheduling script was developed to preferentially direct these patients towards the preoperative clinic. We measured clinic fill rates to assure success. This script was provided for all patients without exclusions or alternative pathways based on patient factors, co-morbidities or surgery type.

PDSA cycle 4 focused on designing and implementing a templated EHR tool. This tool provides embedded decision support to guide providers through recommended documentation format, orders, patient instructions, and billing to be completed during a preoperative encounter. It was our hope that the smart-set would be used by clinicians outside of the preoperative clinic and wide adoption could drive improved rates of appropriate testing throughout our division.

EpicTM (EPIC Corporation, Verona Wisconsin) smartsets provide a customizable menu of options including various documentation templates, pre-populated orders, follow-up instructions, and billing codes to promote a more standardized approach to these encounters. The template was revised and approved by our Institution's Evidence Based Order Set Committee prior to implementation.

Release of the smart-set was initially announced at a division wide meeting and by email. Real time support and feed-back on utilization and improvements to the smart-set were not provided. Unfortunately, end-user use was limited. While several efforts were undertaken to improve usability, it was felt that front-line IT support and a formal venue for feedback on usability would have been required for wider adoption; these resources were unavailable during our project timeline.

It was hypothesized that these PDSA cycles would help us reach our aims reducing the rate of unnecessary preoperative testing across our division, defined by published guidelines, in patients undergoing preoperative assessment from $36 \%$ to $10 \%$ over a 9 month period.

\section{RESULTS}

Our section performed 547 preoperative visits between August 2014 and March 2015, with 201 (37\%) conducted by a dedicated preoperative Associate Provider. Eighty-five $(16 \%)$ clinic patients who presented for preoperative care received unnecessary testing during this period. Only 7 of the 201 patients $(4 \%)$ seen in our intervention group had unnecessary testing ordered while 78 patients (23\%) receiving usual care had at least one unnecessary preoperative test ordered. When rates between the two groups were analyzed using Analysis of Means differences between the two groups were statistically significant. When analyzed by Chi Square, results remained significantly different $(\mathrm{P}<.001)$. Mean scheduled appointment duration was also significantly lower in the preoperative clinic compared with the usual care group ( 48 minutes vs. 40 minutes, $\mathrm{P}$ value $=0.014$ by student's t-test). 
Per our initial aims, we also tracked data for the entire division, which included the intervention group of patients. While comparison between the intervention group and the usual care group showed a significant decrease in rates of unnecessary preoperative testing, time ordered data for the entire division did not show a statistically significant change during the study period (Figure 1).

\section{LESSONS AND LIMITATIONS}

We demonstrated that an Associate Provider staffed preoperative clinic within a GIM division can reduce rates of unnecessary testing and time of scheduled appointments. While our project was successful in establishing a dedicated preoperative clinic, we did not demonstrate changes in testing rates across the entire division as we initially aimed. One of the main challenges to fully realizing the potential of this intervention was the difficulty in reliably filling the allotted clinic time with preoperative visits. Specific challenges related to scheduling included concurrent system wide scheduling re-design, turnover of scheduling staff personnel, and the ability of patients to request being seen by their PCP instead of in the preoperative clinic. These scheduling barriers are likely shared across many primary care settings.

Scheduling scripts were revised towards a more directive template with an "opt out" mechanism, ultimately providing the best fill rates. Achieving acceptable fill rates in the intervention clinic requires direct outreach to the scheduling staff, routine educational sessions and regular surveillance of fill rates. At times, other care needs such as acute visits were prioritized, and preoperative clinic visits were dispersed to usual care. We feel that if a scheduling scheme could reliably assign a majority of preoperative patients to the preoperative clinic we would have been successful in decreasing the overall rates of unnecessary testing below 10 percent.

Another challenge for our preoperative clinic was preserving continuity between patients and their providers. Having Associate Providers perform the preoperative exam ran counter to other scheduling efforts in our department attempting to prioritize primary care continuity in certain patients. Valid institutional priorities to improve continuity should be carefully balanced against protecting clinician time to trial innovative care models like the preoperative clinic.

We were initially hopeful that the introduction of the EHR smart set would drive the largest change of preoperative testing rates across the entire clinic, and not merely within the preoperative clinic. That this expectation was not realized provided a valuable learning opportunity. Previously there had not been a strong culture of EHR innovation and no prior use of encounter templates in day-to-day clinical work within our department. As such, the process of using this template was sufficiently different from normal documentation workflow that many providers did not wish to or were not able to implement it into their workflow.

The time and face-to-face support necessary for adoption of this type of tool is likely quite variable, and ideally would be made available in real-time. Our

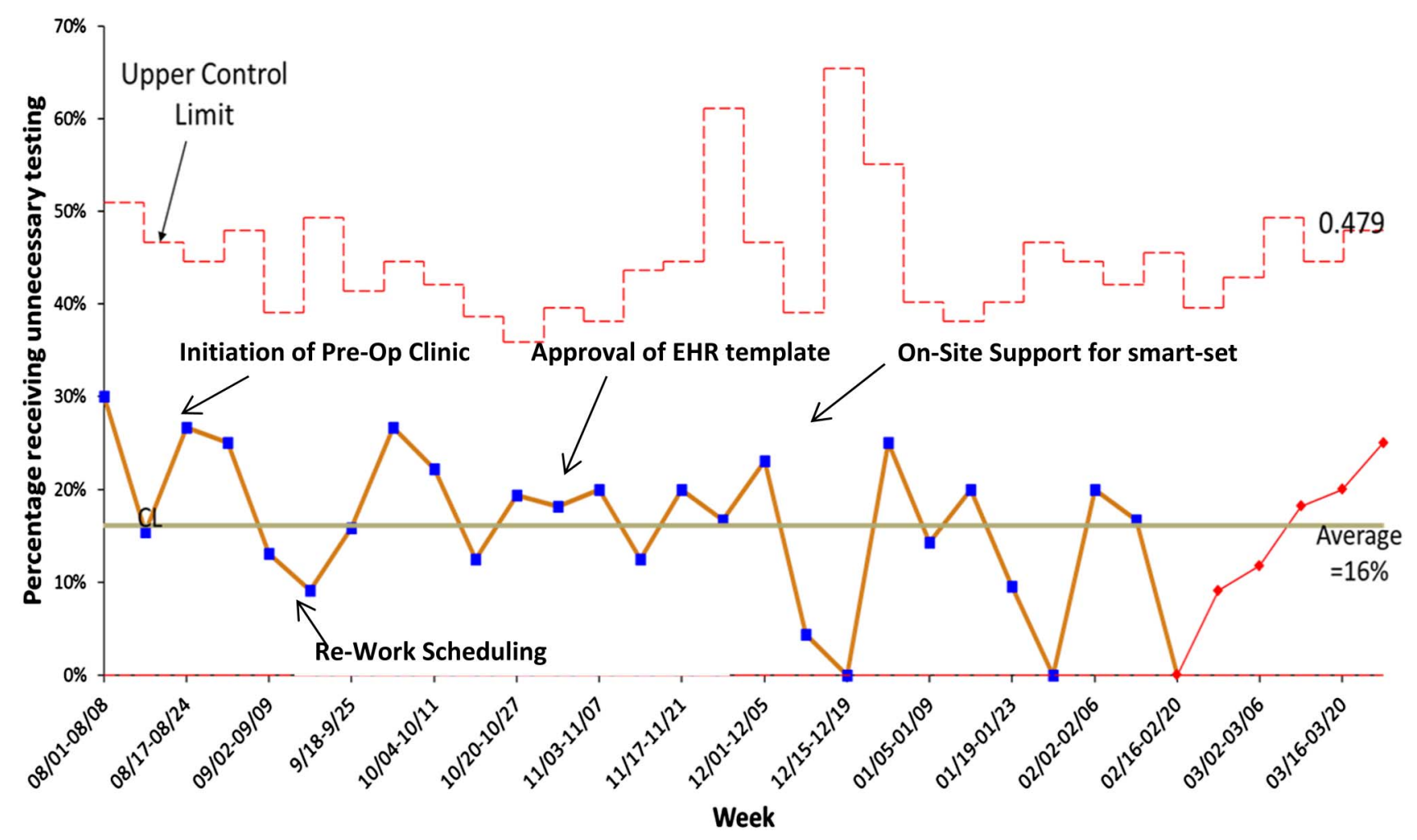

Results: P-Chart of All GIM patients receiving unnecessary testing during intervention period, August 2014-March 2015 
experience was that just providing the tools along with a brief educational session was not sufficient to drive performance improvement. In the future, EHR tool implementation should be accompanied by more intensive, real-time support with regular follow-up of provider usage and concerns.

Further work is needed to adopt this type of intervention on a larger scale and in a different setting as the intervention was piloted at a single academic medical center. The preoperative evaluations in our section are only performed on patients undergoing low and intermediate cardiac risk surgeries, however, this lower complexity allowed for clearer distinctions in testing protocols and in determination of whether a test was considered unnecessary. The relative low complexity of these visits also allowed a focus on efficiency which may not be possible in settings where higher complexity preoperative evaluations are also being performed. Other clinics where a wider breadth of preoperative care is provided may need to structure their interventions differently. Lastly, we studied mean scheduled appointment time and not actual visit time. While we think we reduced actual visit time, this was not specifically measured.

There are some additional considerations related to the generalizability of our approach. As an academic practice, most providers do not practice full time and patients are accustomed to not necessarily seeing their primary care provider for all visit types. This model may have led to less resistance from PCPs and patients compared to a practice where a higher percentage of PCPs are engaged in full-time clinical practice. Some institutions may be constrained by rigid rules that require certain preoperative testing prior to surgery. Also, as a part of the Medicare Pioneer Accountable Care Organization (ACO) we had a high proportion of elderly patients enrolled in global payment models which may have separately discouraged routine preoperative testing through other mechanisms.

\section{CONCLUSION}

Compared to usual preoperative care with a PCP we found that a structured preoperative clinic significantly reduced rates of unnecessary preoperative testing while simultaneously improving efficiency. Using Associate providers, there was a 5-fold reduction in unnecessary testing and a $20 \%$ decrease in visit time for preoperative evaluations. Identification of appropriate patients for these clinics and balancing the need for PCP continuity will be an important challenge moving forward.

Acknowledgements Ellen Eisenberg MD, Team Leader Dartmouth-Hitchcock Medical Center Division of General Internal Medicine

Shanon Bean, Practice Manager at Dartmouth-Hitchcock Medical Center

Division of General Internal Medicine

Kellie Yacullo, Scheduling supervisor and team member,

Dartmouth-Hitchcock Medical Center

Daniel Moran APRN, project team member and preoperative clinic staff

Anne-Marie Lorefice PA-C, Project Team member and preoperative clinic staff

Maria Ciambra, Scheduling supervisor and team member,

Dartmouth-Hitchcock Medical Center Division of General Internal Medicine

Declaration of interests nothing to declare

Ethical approval The Dartmouth College Institution's Institutional Review Board (IRB) deemed that this project was a "quality improvement project without a research component" and was exempt from IRB review.

Open Access This is an open-access article distributed under the terms of the Creative Commons Attribution Non-commercial License, which permits use, distribution, and reproduction in any medium, provided the original work is properly cited, the use is non commercial and is otherwise in compliance with the license. See:

- http://creativecommons.org/licenses/by-nc/2.0/

- http://creativecommons.org/licenses/by-nc/2.0/legalcode

\section{REFERENCES}

1. Berwick DM, Hackbarth AD. Eliminating waste in US health care. Jama 2012;307:1513-1516.

2. Benarroch-Gampel J, Sheffield KM, Duncan CB, et al. Preoperative laboratory testing in patients undergoing elective, low-risk ambulatory surgery. Annals of surgery 2012;256:518-528.

3. Center for Medicare and Medicaid Services. US Code of Federal Regulations- State Operations Manual Appendix A. CMS; 2013 [cited 2014 11/21]; §482.52 (c) (5) A-0952: Available from: https:// www.cms.gov/Regulations-and-Guidance/Guidance/Manuals/ downloads/som107ap_a_hospitals.pdf.

4. Delahunt B, Turnbull PR. How cost effective are routine preoperative investigations? The New Zealand medical journal 1980;92:431-432.

5. Hepner, David L., Angela M. Bader. "If I Had a HammerBuilding Alignment and Accountability." The Journal of the American Society of Anesthesiologists 124.4 (2016): 755-757.

6. Sigmund AE, Stevens ER, Blitz JD, Ladapo JA. USe of preoperative testing and physicians' response to professional society guidance. JAMA Internal Medicine 2015;175:1352-9.

7. Apfelbaum JL, Connis RT, Nickinovich DG, et al. Practice advisory for preanesthesia evaluation: an updated report by the American Society of Anesthesiologists Task Force on Preanesthesia Evaluation. Anesthesiology 2012;116:522-538.

8. The American Board of Internal Medicine Foundation. Choosing Wisely- Clinician Lists. Philadelphia, PA: The ABIM Foundation; 2016 [cited 2016 5/21/16]; Available from: http://www.choosingwisely. org/clinician-lists/\#keyword=preoperative.

9. Nelson EC, Batalden PB, Godfrey MM. Quality by design. A clinical microsystems approach. 2007. 